Original article

\title{
FEMORAL LENGTHENING USING MODIFIED LOW PROFILE ILIZAROV FIXATOR IN ADOLESCENTS: DESCRIPTION OF THE TECHNIQUE AND EARLY EXPERIENCE
}

\author{
Abdel Rahman Hafez ${ }^{(*)}$, Ashraf Marzouk, Wael Salama, Mohamed Ali, Mohamed Kenawey
}

Orthopedic Surgery dept., Faculty of Medicine, Sohag University, Sohag, Egypt

*E-mail: dr.abdelrahman_alshikh@yahoo.com

\begin{abstract}
We modified the original Ilizarov frame described for femoral lengthening and used two full rings fixed by 3 tensioned wires for each with an osteotomy in the distal metaphyseal area. Four femoral lengthenings were performed and followed up for at least 30 months. Average age of our patients was 15 years and length gain was $74 \pm 18 \mathrm{~mm}$ with one patient had concomitant correction of valgus deformity. The planned lengthening was achieved in all patients with healing index $19 \pm 2$ days/cm. Advantages for this low profile frame are mainly less bulky external fixator and fewer transosseous elements and therefore pin site problems.
\end{abstract}

Keyword: Femoral lengthening, Ilizarov lengthening, Pediateric leg lengthening, Low profile external fixator, femoral deformity

\section{Introduction}

The first report of successful lengthening technique was by Codivilla [1] in 1905, using an oblique osteotomy followed by powerful acute traction through a calcaneal pin. In 1913, Ombredanne [2] was the first to use an external fixator for limb lengthening. He lengthened the femur gradually in a rate of $5 \mathrm{~mm} /$ day for 8 days achieving $4 \mathrm{~cm}$ of lengthening. Early $1950^{\text {th }}$ was the start of the revolutionary contributions of Gavril Ilizarov in limb lengthening surgery and distraction osteogenesis using his circular external fixator [3-5]. Since that time, different forms of external fixators have been used in limb lengthening and reconstruction [6-8]. External fixators used specifically in femoral lengthening are bulky and non comfortable for the patients $[9,10]$. With the widespread use of different lengthening devices and techniques, many modifications were described to make the lengthening procedure a good experience and as comfortable as possible. All of these modifications aimed at either eliminating the use of external fixation completely through the use of totally implantable intramedullary lengthening nails or trying to shorten the external fixation time [10-13]. Three modifications were described to shorten the external fixation time during femoral lengthening in pediateric population and adolescents; lengthening over intramedullary humeral nail [14], lengt- 
hening with submuscular locked plate stabilization [15] or lengthening over elastic stable intramedullary nails [16], however deep infection is a significant risk in all of these techniques. We had modified the original Ilizarov frame which is usually used for femoral lengthening to make it less bulky. Two full rings were used and fixed to the bone by 2 tensioned wires and a drop out wire from each ring. The frame was placed in the distal part of the

\section{Patients and methods}

\subsection{Rational}

Circular Ilizarov external fixators used in femoral lengthening usually consist of two full rings distally and one or two proximal arches, or one distal full ring, middle half ring and a proximal arch $[10,17,18]$. This proximal arch extends the fixation to the proximal femur using half pins. Lengthening frames usually gain substantial stability with progressive distraction through the resistance of the soft tissue envelop. Therefore, a shorter ring block can be used for fixation with only one ring in each bone segment fixed by multiple k-wires in different planes [19]. This was Ilizarov's original description of lengthening through two corticotomies performed at both the proximal and distal ends of the bone being elongated. During

\subsection{Patients' demographics}

Approval of the local ethics review board was obtained. Four femora in 3 patients were lengthened using this technique. Average age of our patients at the time of lengthening was $15 \pm 2.7$ years (range, 11-17 years). All patients were followed up for at least 30 months. The etiology of shortening was malunited neck femur with coxa vara $(n=1)$, poliomyelitis with genu valgum deformity and shortening

\subsection{Operative technique}

Operative procedures were performed with the patient in supine position on a standard radiolucent orthopaedic table. Smooth k-wires $1.8 \mathrm{~mm}$ in diameter were all the hardware used for frame application. Under the control of image femur and lengthening was through distal metaphyseal osteotomy. This modification has the potential of:

a) Less cumbersome lengthening fixator with better patient satisfaction and experience

b) Providing enough mechanical stability to give an adequate mechanical support for the distraction gap with no risk of axial deviations.

this procedure of bifocal lengthening, each bony segment is actually stabilized by one full ring or one arch and multiple tensioned k-wires [3]. We followed the same concepts and used two full rings in the area of the distal femur with a distal metaphyseal lengthening osteotomy. The originally described proximal arches were not used and therefore no proximal extension for the frame into the proximal femur. Each ring was stabilized using 2 tensioned wires and a third drop out wire. As a result, each bony segment was stabilized by a ring block composed of one full ring and three points of fixation (3 tensioned wires) per ring which are spread in different planes and levels.

$(\mathrm{n}=1)$ and achondroplasia in a patient who had bilateral cosmetic lengthening. Length gain was $74 \pm 18 \mathrm{~mm}$ (range, $50-90 \mathrm{~mm}$ ). Our patient with shortening due to poliomyelitis had associated valgus deformity of $23^{\circ}$ which was mainly femoral. Correction of this deformity using the same frame followed by lengthening of 50 $\mathrm{mm}$ was performed.

intensifier, two wires were drilled through the femoral condyle and attached to the first ring. The first $\mathrm{k}$-wire was driven from just anterior to the posterolateral corner of the lateral femoral condyle, in a posterolateral to anteromedial direction, 
parallel to the knee joint line in the AP projection and strictly within the distal femoral epiphysis. Rubber stoppers were applied to both ends of the wire. An Ilizarov ring of suitable diameter was mounted to the wire and secured using two slotted bolts. The wire was tensioned to $130 \mathrm{~kg}$. A second wire with the same diameter was drilled on the same ring, at an angle of about $60^{\circ}$ to the first wire from anterolateral to posteromedial directions and strictly in the epiphysis and was also secured and tensioned in the same way. A drop out wire was then drilled in a transverse direction using two male posts attached to the first ring just above the physeal line, fig. (1). In case of femoral lengthening, 4 threaded rods were used to attach a second Ilizarov ring 10-12 cm above the first one. Two wires were directly drilled on the second ring, in the same planes as done in the first ring, and secured and tensioned. Using two male posts secured to the second ring, a drop out wire was drilled in a transverse direction just below the ring, fig. (1). Regarding our patient who had $23^{\circ}$ valgus deformity with shortening, the construct was modified to allow for correction of angulation firstly and then lengthening. The two Ilizarov rings were connected to each other using 2 universal hinges and a threaded motor rod (instead of 4 threaded rods used for lengthening only). The two hinges were adjusted intraoperatively to lie opposite the medial femoral cortex at the level of the osteotomy (one anterior hinge and one posterior) in order to allow for a first stage of correction of the valgus deformity, fig $g_{s}$. (2 \& 3). Through 2-3 cm lateral incision, a transverse multiple drill hole subperiosteal osteotomy was performed just above the distal drop-out wire using $3.2 \mathrm{~mm}$ drill bit and $10 \mathrm{~mm}$ osteotome. The two rings were then disconnected and twisted against each other to complete the osteotomy. They were reconnected and a compression of 2-3 $\mathrm{mm}$ was applied to the osteotomy site. The wound was closed in layers and the knee was moved through the maximum range allowed by the Ilizarov rings.

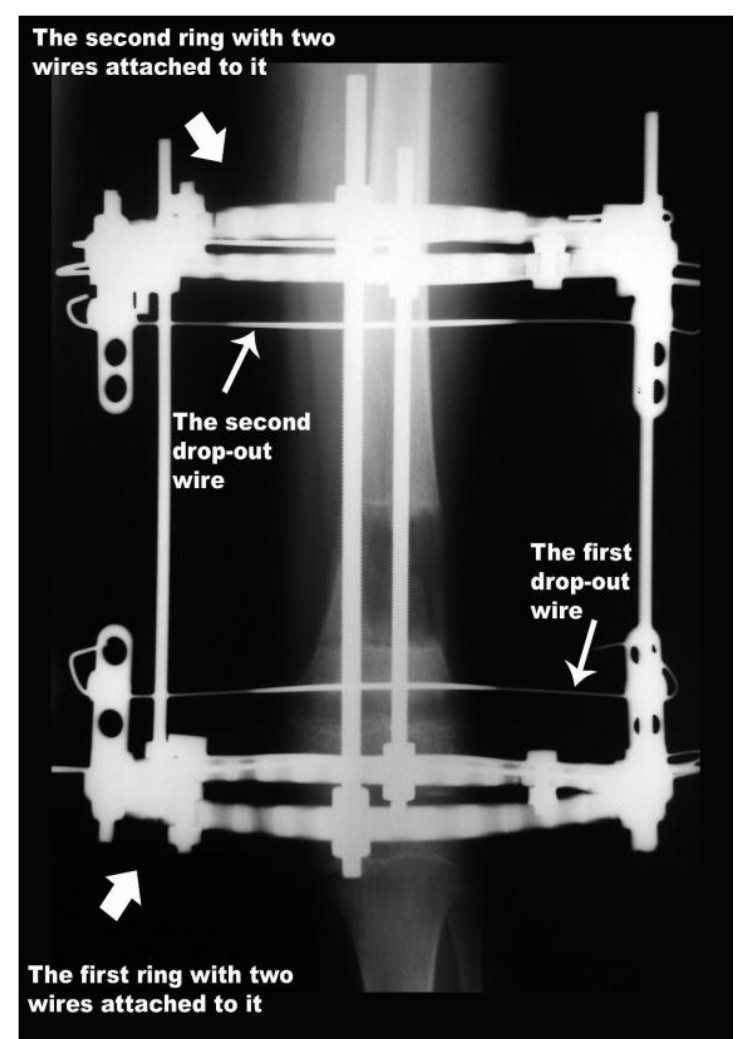

Figure (1) Shows $13^{\text {th }}$ weeks follow up radiograph for a patient with lengthening of $7 \mathrm{~cm}$ using this low profile Ilizarov tec-hnique. 


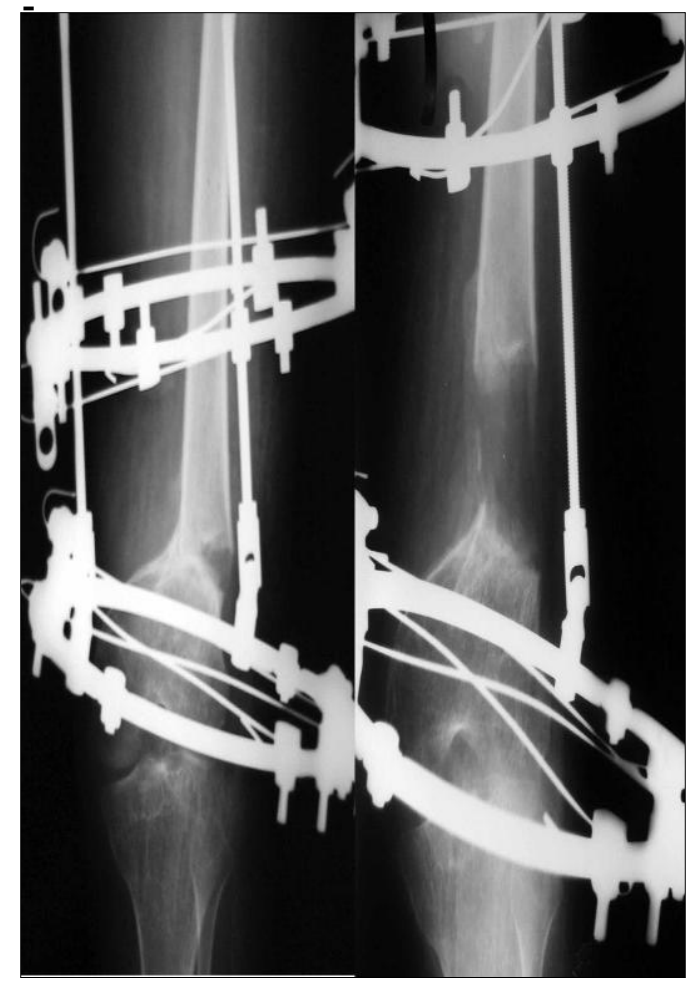

Figure (2) Shows 17 year old pat-ient with valgus deformity $23^{\circ}$ and shortening of $5 \mathrm{~cm}$ due to poliomyelitis. First stage was correction of the valgus deformity (left) and then the second stage was lengthening using the same frame (right).

\subsection{Postoperative workup}

Postoperatively, patients were allowed touchdown weight bearing as tolerated on crutches from the first postoperative day on. Postoperative physiotherapy program consisted of active range of motion exercises of the knee as allowed by the frame together with strengthening exercises for the quadriceps and hamstrings. Patients were discharged from the hospital with careful instruction about daily pin site care. Lengthening started from the $7^{\text {th }}$ postoperative day at a rate of $0.25 \mathrm{~mm} /$ 6 hours. For the patient, who had combined valgus deformity and shortening, a first stage with pure correction of the valgus deformity was performed in a rate of $0.25 \mathrm{~mm} / 6$ hours and its duration was 14 days. Afterwards, the mechanical axis was checked with a full length standing radiograph and then the hinges were locked in this position and the second stage of pure lengthening was started at the same rate, fig s. $(2 \& 3)$. Follow up

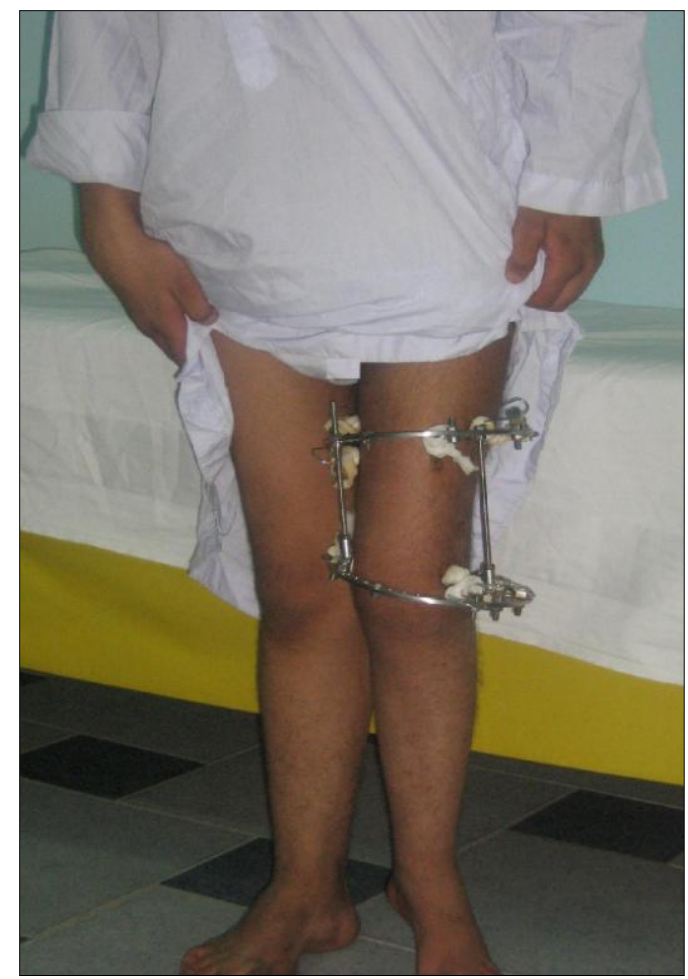

Figure (3) Shows photo for the patient with the device

radiographs were obtained weekly during the distraction phase and then every 4-6 weeks during the consolidation phase. Radiographic consolidation was defined by the presence of 3 continuous cortices across the distraction gap in the AP and Lat views. The frame was then removed gradually, over a period of 4 weeks, with removal of one k-wire from either sides every week, till only 2 wires were remaining, one in the proximal and the other in the distal fragments and then the frame was completely removed. During this gradual removal, patients were instructed to fully weight bear on the extremity. With complete removal of the frame, a post-removal follow up radiograph was requested and then patients were followed up every 3 months, figs. (4 \& 5). An extensive physiotherapy program for the range of the motion of the knee joint as well as the strength of the muscles was performed. 


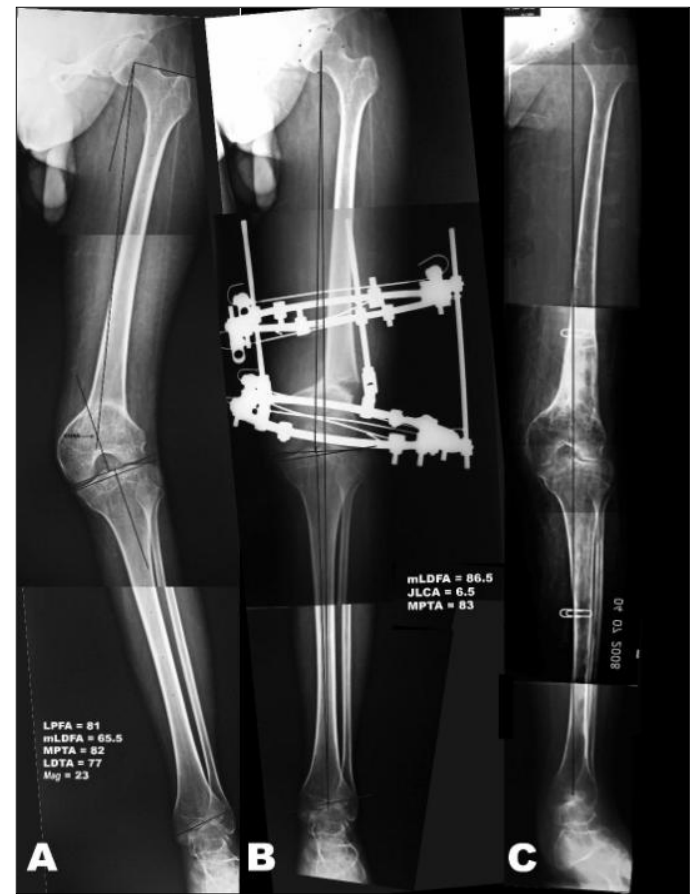

Figure (4) Shows the same patient who had poliomyelitis, shortening of $5 \mathrm{~cm}$ and valgus deformity $23^{\circ}$; $\underline{\text { a. }}$ preoperative full standing radiograph showing a valgus deformity of $23^{\circ}$ with a CORA located at the distal femur, $\underline{\mathbf{b}}$. fourteen days from the start of the first stage correcting the valgus deformity with restoration of the mechanical axis of the limb within the normal range, $\underline{\mathbf{c}}$. postremoval full length standing radiograph with the mechanical axis of the limb passing through the midline of the tibial plateau

\section{Results}

The follow up of our patients was in average $31.5 \pm 1.7$ months (range, 30-34 months). The desired lengthening was achieved in all patients. The healing index of our patients was $19 \pm 2$ days/cm (range, 15-29 days $/ \mathrm{cm}$ ). External fixator time was $25 \pm 5$ weeks (range, 19-31 weeks) and the mean external fixation index was $25 \pm 8$ days/cm (range, 19-37 days/cm). Superficial pin track infections (grade I according to Paley classification [20] occurred in all patients and they responded well to local pin site care and oral antibiotics. In no instance did we have to remove or change a wire. No cases of persistent infection following removal of the frame. The ranges of motion of the

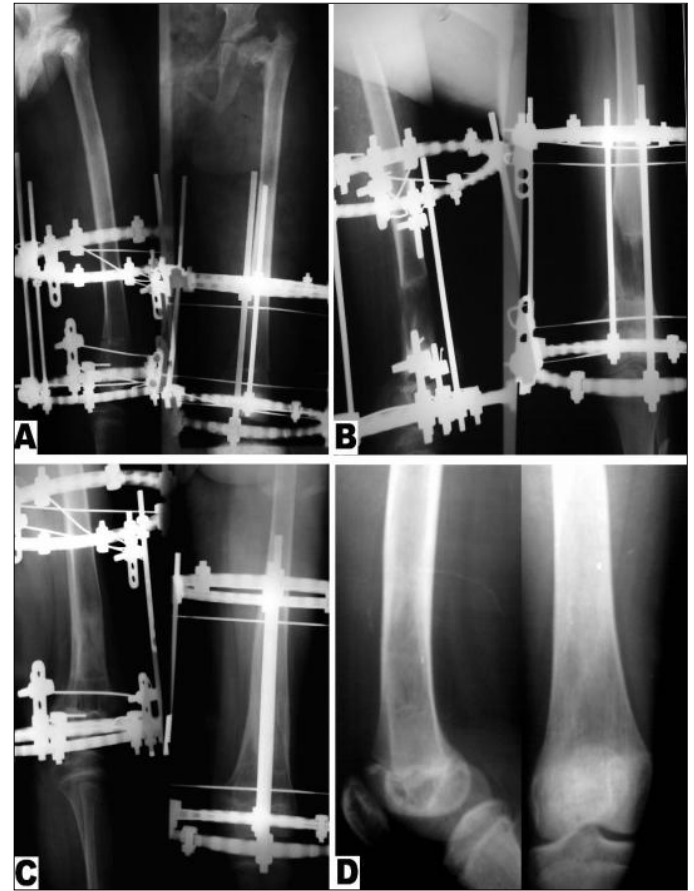

Figure (5) Shows eleven years old patient with shortening caused by malunited old femoral neck fracture and coxa vara, a. postoperative radiograph with the frame in place and a distal femoral osteotomy, $\underline{\mathbf{b}}$. follow up radiograph 13 weeks postoperatively after lengthening of $7 \mathrm{~cm}$ and the distraction gap shows a good regenerate, $\underline{\text { c. follow }}$ up radiograph 19 weeks postoperatively with fully consolidated regenerate filling the distraction gap, $\underline{\mathbf{d}}$. follow up radiograph 27 months after removal of the frame showing complete restitution of the medullary cavity with very good alignment.

hip and ankle joints were free at the latest follow up. The knee joint range of motion was $140^{\circ}$ and $130^{\circ}$ of flexion with full extension in the two patients who had unilateral femoral lengthening. The patient with achondroplasia and bilateral cosmetic femoral lengthening had full extension of the knees while flexion was $110^{\circ}$ and $70^{\circ}$ on the left and right sides respectively. No cases of intraoperative complications or iatrogenic neurovascular injury were present. No cases of axial deviation whether in the frontal or the sagittal plane or refracture of the newly formed regenerate following frame removal occurred. 


\section{Discussion}

Femoral lengthening in pediateric patients and adolescents is usually performed using different forms of external fixators whether monolateral or circular $[7,8,10$, 21,22]. All circular fixators which are used in femoral lengthening are bulky and may cause significant patient discomfort and even psychological problems which sometimes necessitate cessation of lengthening $[9,10,23]$. Advantages of our technique other than a less bulky fixator are firstly, the lengthening osteotomy is made in a metaphyseal area. Metaphyseal tibial lengthening was shown by Fischgrund et al. [21] to heal significantly faster than diaphyseal lengthening. In an animal study for Aronson et al. [24], metaphyseal coritcotomies healed earlier than the diaphyseal corticotomies, however this was not statistically significant. Furthermore, using quantitative computed tomography to evaluate the density of the newly formed regenerate, statistically significant higher densities were found for metaphyseal regenerate. Second, the only transosseous elements used for fixation of our frame were $6 \mathrm{~K}$-wires, $1.8 \mathrm{~mm}$ in diameter in a multiplanar fashion through a circular external fixator. No fixation by Schanz screws as done in monolateral external fixators which is biomechanically a cantilever method of fixation. Circular external fixator with only tensioned wires provides the ideal mechanical environment for distraction osteogenesis and bone healing [25]. Tensioned wires also cause minimal pin tract infection which resolve rapidly after removal of the frame [26,27]. Moreover, the frame was stable enough to support the newly regenerated bone evidenced by the absence of any axial deviation even in the case with concomitant correction of valgus deformity. Also, no refractures of the newly formed regenerate following fixator removal took place. The patient with achondroplasia who underwent bilateral femoral lengthening had unfavorable knee range of motion. This patient was not compliant with the postoperative rehabilitation protocol and even with the post fixator removal physiotherapy program. Non-compliance is a major concern with all forms of external fixators used in limb lengthening [26,27]. However, all of our patients were satisfied with the final result of treatment. Several types of fully implantable lengthening nails were recently developed, however due constrains of size and length of the nails, they are mainly used in adults. One report discussed the use of the motorized lengthening nail (Fitbone) in 8 preadolescent patients ( $>13$ years) [28]. However, this report was biased by the specific selection criteria for this procedure excluding all patients with history of deep infection or chronic osteomyelitis within the last two years or patients with instability of adjacent joints, extension deficit of the knee or equinus deformities. Additionally, technical problems within the nail were reported in this series with one nail failure and one nail breakage and therefore the planned lengthening was not achieved in 2 patients. Other limitations are the cost and the availability because this nail is available only in centers certified by the manufacturer $[9,28]$. Another approach was to decrease the external fixation time through lengthening over intramedullary nails or locked plates. Gordon et al. [14] reported 9 cases of femoral lengthening in children using monolateral external fixator over a humeral nail. They approached the medullary canal through a lateral entry point within the greater trochanter to avoid entry through the piriformis fossa which is associated with the risk of avascular necrosis of the femoral head. However the risk of destroying the trochanteric apophysis with subsequent development of femoral neck valgus cannot be avoided. All of their patients had superficial pin tract infection with two deep infections and osteomyelitis. Three patients had fractures, one through the site of distal half pin three months after frame removal and two through the distal locking of the nail. Another combination for lengthening over an intra- 
medullary fixation was described by Lampasi et al [16]. They used elastic stable intramedullary nails with monolateral external fixator for femoral lengthening in 6 patients with mean age at surgery 6.6 years. The external fixators were left in place throughout the distraction and consolidation phases which contradicted one of the main proposed aims and advantages of the technique. Iobst et al. [15] described a technique for femoral lengthening over locked submusclar plate using a standard Ilizarov frame in 6 children with average age 7.6 years. Their frame consisted of femoral arch(es) proximally connected to full ring and/or 5/8 ring. They reported 7 complications in 6 patients. Serious complications included premature consolidation, a translational deformity of the regenerate and apex anterior angulation, and a fracture at the superior edge of the locking plate caused by a fall 3 months following removal of the fixator. Severe complications were 11 and $18^{\circ}$ procurvatum deformity. Moreover, deep infection remains a significant risk with this technique.

\section{Conclusion}

The search for modifications to achieve better patients' compliance and easier lengthening technique is always going on. Our technique can be added to the armamentarium of these modifications; however a larger series of patients is required to prove the safety and better patients' experience.

\section{References}

[1] Codivilla, A. (1905). On the means of lengthening, in the lower limbs, the muscles and tissues which are shortened through deformity. American J Orthop Surg. 2: 353-369.

[2] Ombredanne, L. (1913). Allongement d'un femur sur un membre trop court. Bull Mem Soc Chirur (Paris). 39: 1177-1180.

[3] Ilizarov, G. (1990). Clinical application of the tension-stress effect for limb lengthening. Clin Orthop Relat Res. 250: 8-26.

[4] Ilizarov, G. (1989). The tension-stress effect on the genesis and growth of tissues: part I. The influence of stability of fixation and soft-tissue preservation. Clin Orthop Relat Res. 238: 249-81.

[5] Ilizarov, G. (1989). The tension-stress effect on the genesis and growth of tissues: part II. The influence of the rate and frequency of distraction. Clin Orthop Relat Res. 239: 263-285.

[6] Catagni, M., Lovisetti, L., Guerreschi, F., et al. (2005). Cosmetic bilateral leg lengthening: Experience of 54 cases. J Bone Joint Surg Br. 87: 10: 14021405.

[7] De Bastiani, G., Aldegheri, R., Renzi-
Brivio, L., et al. (1987). Limb lengthening by callus distraction (callotasis). J Pediatr Orthop. 7 (2): 129-134.

[8] Wagner, H. (1978). Operative lengthening of the femur. Clin Orthop Relat Res. 136: 125-142.

[9] Simpson, A., Shalaby, H., Keenan, G. (2009). Femoral lengthening with the Intramedullary skeletal kinetic distractor. J Bone Joint Surg Br. 91-7: 955-961.

[10]Paley, D., Herzenberg, J., Paremain, G., et al. (1997). Femoral lengthening over an intramedullary nail. A matchedcase comparison with Ilizarov femoral lengthening. J Bone Joint Surg Am. 79 (10): 1464-1480.

[11]Betz, A., Baumgart, R., Schweiberer, L. (1990). First fully implantable intramedullary system for callus distraction--intramedullary nail with programmable drive for leg lengthening and segment displacement. Principles and initial clinical results. Chirurg. 61 (8): 605-609.

[12]Cole, J., Justinm D., Kasparis, T., , et al. (2001). The intramedullary skeletal kinetic distractor (ISKD): First clinical results of a new intramedullary nail 
for lengthening of the femur and tibia. Injury. 32 (Suppl 4): SD129SD139.

[13] Guichet, J., Casar, R. (1997). Mechanical characterization of a totally intramedullary gradual elongation nail. Clin Orthop Relat Res. 337: 281-290.

[14]Gordon, J., Goldfarb, C., Luhmann, S., et al. (2002). Femoral lengthening over a humeral intramedullary nail in preadolescent children. J Bone Joint Surg Am. 84 (A-6): 930-937.

[15]Iobst, C., Dahl, M. (2007). Limb lengthening with submuscular plate stabilization: A case series and description of the technique. J Pediatr Orthop. 27 (5): 504-509.

[16]Lampasi, M., Launay, F., Jouve, J., et al., (2009). Femoral lengthening over elastic stable intramedullary nailing in children using the monolateral external fixator. Chir Organi Mov. 93 (2): 57-64.

[17]Catagani, M., Ottaviani, G. (2008). Ilizarov method to correct limb length discrepancy after limb-sparing hemipelvectomy. J Pediatr Orthop B. 17 (6): 293-298.

[18]Solomin, L. (2008). The basic principles of external fixation using the Ilizarov device. $1^{\text {st }}$ ed., Springer-Verlag Italia,. Milan-Italy

[19]Fragomen, A., Blyakher, A., Ilizarov, S. (2007). Mechanical principles of the Ilizarov method. In: Robert Rozbruch, S., Ilizarov, S., (eds.) Limb lengthening and reconstruction surgery, Informa Healthcare, NY.:19-42.

[20]Paley, D. (1990). Problems, obstacles, and complications of limb lengthening by the Ilizarov technique. Clin Orthop Relat Res. 250: 81-104.
[21]Fischgrund, J., Paley, D., Suter C. (1994). Variables affecting time to bone healing during limb lengthening. Clin Orthop Relat Res. 301: 31-37.

[22]Paley, D. (1988). Current techniques of limb lengthening. J Pediatr Orthop. 8 (1): 73-92.

[23]Stanitski, D., Bullard, M., Armstrong, P., et al. (1995). Results of femoral lengthening using the Ilizarov technique. J Pediatr Orthop. 15 (2): 22431.

[24]Aronson, J. (2007). Basic science and biological principles of distraction osteogenesis. In: Robert Rozbruch, S., Ilizarov, S, (eds.) Limb lengthening and reconstruction surgery, Informa Healthcare, NY.: 19-42.

[25]Stein, H., Perren, S., Mosheiff, R., et al. (2001). Decline in transfixing $\mathrm{K}$-wire tension of the circular external fixator: Experimental continuous in vivo measurements. Orthopedics. 24 (10): 985-989.

[26]Schwartsman, V. (1995). Circular external fixation in high tibial osteotomy. Instr Course Lect. 44: 469474.

[27]Shiha, A., Alam El-Deen, M., Khalifa, A., et al. (2009). Ilizarov correction of genu varum deformity in adults. Acta Orthop Belg. 75 (6): 784-791.

[28] Krieg, A., Speth, B., Foster, B. (2008). Leg lengthening with a motorized nail in adolescents: An alternative to external fixators? Clin Orthop Relat Res. 466 (1): 189-197. 